\title{
Measurement of UV-induced absorption and scattering losses in photosensitive fibers
}

\author{
X. Roselló-Mechó, M. Delgado-Pinar ${ }^{*}$, J. L. Cruz, A. Díez, and M. V. Andrés \\ ${ }^{1}$ Department of Applied Physics and Electromagnetism-ICMUV, University of Valencia, C/ Dr. Moliner 50, Burjassot 46100, Spain. \\ *Corresponding author: martina.delgado@uv.es
}

UV-assisted fabrication of gratings using photosensitive fibers is a well-established technique, based on the UV-induced permanent modification of the refractive index of the fiber material. As a result, the absorption coefficient is also changed. Here, we exploit the thermal sensitivity of whispering gallery modes resonances of the fiber itself to measure the profile of the heating along an irradiated fiber versus the input power and for different UV radiation intensities. Our technique allows discriminating between the absorption and scattering contributions to the overall losses, by comparing the results obtained with our technique with direct transmission measurements. Different photosensitive fibers are characterized by means of this technique. Non uniform UV irradiation of photosensitive fibers can be characterized with this technique. ๑ 2018 Optical Society of America

OCIS codes: (060.2370) Fiber optics sensors; (060.2270) Fiber characterization; (060.2300) Fiber measurements; (060.3738) Fiber Bragg gratings, photosensitivity; (240.6690) Surface waves.

UV-assisted inscription of fiber Bragg gratings (FBGs) and long period gratings (LPGs) is currently the most employed method for their fabrication. Typically, a photosensitive (PS) fiber is exposed to a UV patterned beam that modulates permanently the refractive index of the core [1]. Some gratings with specific features require of considerable amount of UV energy $[2,3]$. The photosensitivity of a given fiber depends on the chemical composition of the core $[4,5]$. Here, we will study a germano-silicate boron codoped fiber, a highly germano-silicate doped fiber, and a hydrogenated conventional telecom fiber. According to Kramers-Kronig relations, the change in the refractive index is associated with a variation of the absorption coefficient. Also, the exposure of the fiber to the energy levels of UV-radiation required for gratings inscription may induce a mechanical deformation in the fiber, such as corrugations due to the densification of the material [6] (one should have in mind that for a grating of $-30 \mathrm{~dB}$ minimum transmittance and $1 \mathrm{~cm}$ long, it is needed about 10 minutes of exposure time in order to get a fluence of $150 \mathrm{~J} / \mathrm{mm}^{2}$ ). This leads to the increment of scattering loss of the gratings. A direct transmission measurement during UV-irradiation provides an average of the attenuation coefficient along the total length of the processed section [7], which includes both contributions, i.e., absorption and scattering. Here, we propose to use the whispering gallery mode (WGM) resonances of the fiber itself to measure the increase of the UV-induced absorption loss. Thus combining both types of measurements, one can evaluate the absorption and scattering contributions to the overall losses.

WGM resonances have been demonstrated to be useful for the characterization of optical fibers: thermal effects in active fibers [8], fiber diameter fluctuations [9, 10], and Pockels' coefficients [11]. The WGM resonances are excited azimuthally using an auxiliary microfiber at a given point of the fiber, which plays the role of the microresonator. The optical wavelength of a given resonance is set by the refractive index of the microresonator's material (silica, in our case) and its diameter, which depend on the temperature. The typical high Q-factor of the resonances, between $10^{6}$ and $10^{7}$, allows achieving very low detection limits (DL) for the temperature variation [8].

First, we will describe the measuring technique. We will use moderate input powers (up to $\sim 1 \mathrm{~W}$ ) in order to induce a measurable temperature variation, $\Delta T$. An increased absorption coefficient will produce a higher heating along the irradiated section, when compared to the pristine fiber section. Moreover, if the section is not uniformly irradiated, it will result in a gradient of temperature along it. The relative wavelength shift of a WGM resonance, $\Delta \lambda / \lambda$, as a function of $\Delta T$ is given by:

$$
\frac{\Delta \lambda}{\lambda}=\left(\frac{1}{R} \frac{d R}{d T}+\frac{1}{n_{\mathrm{eff}}} \frac{d n_{\mathrm{eff}}}{d T}\right) \cdot \Delta T
$$

where $\lambda$ is the central wavelength of the unperturbed resonance, $R$ is the fiber radius, and $n_{\text {eff }}$ is the effective index of the WGM. The first term of the equation is determined by the material thermal expansion coefficient, and the second depends on both the material thermo-optic effect and the fiber radius thermal expansion. Accurate numerical simulations demonstrate that any WGM resonance centered at $1.55 \mu \mathrm{m}$ propagating in a standard optical fiber shifts towards longer wavelengths at the same rate, which was experimentally measured, $8.2 \mathrm{pm} /{ }^{\circ} \mathrm{C}[8]$. This value is valid for different azimuthal modes and for different radius of the resonator (it changes in less than $0.1 \%$ when resonator diameter changes from 100 to $125 \mu \mathrm{m}$ ). Here we 
will assume that the heating over the transversal section of the fiber, at a given axial position, is determined by the absorption coefficient, $\alpha^{\text {abs }}$ [12]. Following the analysis developed in this reference, we find that the steady state value of $\Delta T$, for a given input power $P$, follows the expression:

$$
\frac{\Delta T}{P}=\frac{1}{2 \pi b h} \alpha^{\mathrm{abs}}
$$

where $b$ is the fiber radius and $h$ is the heat transfer coefficient (for standard fiber: $b=62.5 \mu \mathrm{m}$ and $h=81.4 \mathrm{~W} \cdot \mathrm{m}^{-2} \cdot \mathrm{K}^{-1}[12]$ ). Thus, the ratio of $\Delta T$ at two different positions along a fiber, 1 and 2 , is proportional to the ratio of $\alpha^{\text {abs: }}$

$$
\frac{\Delta T_{2}}{\Delta T_{1}}=\frac{\alpha_{2}^{\mathrm{abs}}}{\alpha_{1}^{\mathrm{abs}}}
$$

Figures 1(a) and 1(c) show a scheme of the setup employed for the experiments. WGM resonances are excited at a given point using the evanescent field of a $2 \mu \mathrm{m}$ auxiliary tapered fiber, placed perpendicularly to the microresonator. The taper can be swept along the fiber to carry out a thermal characterization [13]. This enables a point to point measurement of the temperature profile with a spacial resolution limited by the axial extension of WGM in cylindrical microcavities, which is about $200 \mu \mathrm{m}$ for a resonance with $\mathrm{Q} \sim 10^{6}$ [14]. A tunable, linearly polarized laser (linewidth $<300 \mathrm{kHz}$ ) was launched to the taper through an optical circulator. The setup allows measuring the WGM resonances in transmission $(\mathrm{T})$ and reflection $(\mathrm{R})$ [13]. The polarization controller (PC) included in the setup permits to excite separately the TE and TM resonances. The setup was isolated to prevent external perturbations.

Figure 1(b) shows a typical reflection spectrum of a WGM resonance obtained with our setup $\left(\lambda_{0}=1540 \mathrm{~nm}\right)$. The $3-\mathrm{dB}$ spectral linewidth is $36 \mathrm{fm}$ (Q-loaded factor of $4 \cdot 10^{7}$ ). If we assume that the resolution of the technique equals the width of the resonance, then the minimum $\Delta T$ that we can measure is $5 \cdot 10^{-3}{ }^{\circ} \mathrm{C}$. However, vibrations and other perturbations that are present in our experimental arrangement limit the DL to $0.03^{\circ} \mathrm{C}$.

(a)
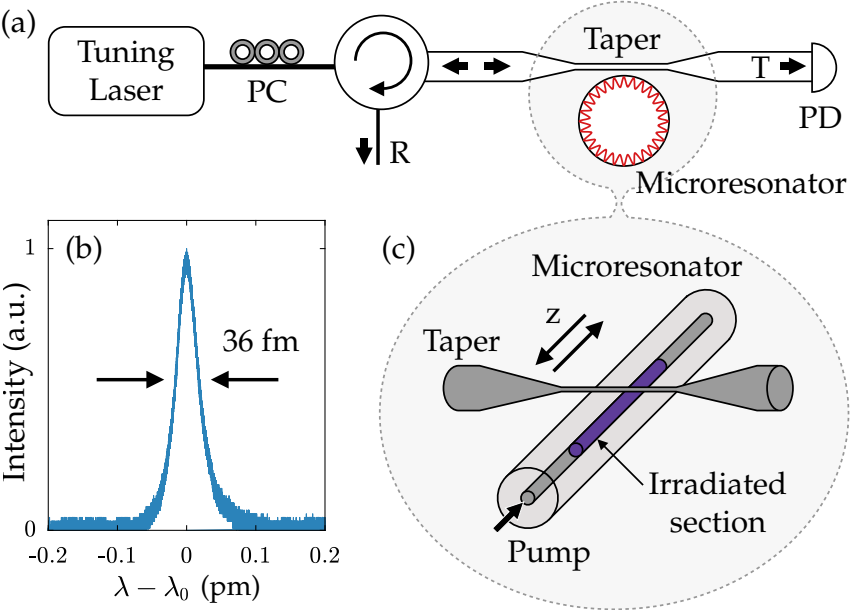

(c)

Microresonator

Fig. 1. (a) Scheme of the setup. (b) Reflection trace of a typical WGM resonance. (c) Detail of the relative position between the irradiated fiber and the microtaper.
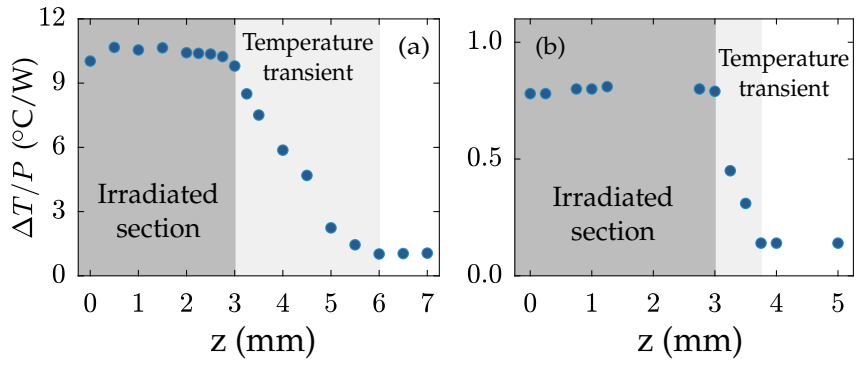

Fig. 2. Typical thermal profile of an irradiated section of PS980. Input signal at (a) $1550 \mathrm{~nm}$, (b) $975 \mathrm{~nm}$.

The thermal profiles of different PS fibers after UV-irradiation were measured. The tested fibers were: (i) Fibercore PS980, $\mathrm{NA} \sim 0.13$, cutoff wavelength $940 \mathrm{~nm}$, attenuation $11.4 \mathrm{~dB} / \mathrm{km}$ at $980 \mathrm{~nm}$; (ii) Fibercore PS1250, NA 0.13, cutoff wavelength $1214 \mathrm{~nm}$, attenuation $131.1 \mathrm{~dB} / \mathrm{km}$ at $1550 \mathrm{~nm}$; (iii) Fibercore SM1500, NA $\sim 0.29$, cutoff wavelength $1387 \mathrm{~nm}$, attenuation $1.95 \mathrm{~dB} / \mathrm{km}$ at $1550 \mathrm{~nm}$; (iv) Corning SMF28, NA 0.14, cutoff wavelength $1260 \mathrm{~nm}$, attenuation $0.188 \mathrm{~dB} / \mathrm{km}$ at $1550 \mathrm{~nm}$; this fiber was hydrogenated for 15 days (pressure: 30 bar) to increase its photosensitivity. A length of each fiber was exposed to a UV fluence of $150 \mathrm{~J} / \mathrm{mm}^{2}$, provided by a doubled-argon laser $(244 \mathrm{~nm})$. The optical signal launched through them to induce heating was provided by two CW laser sources: (a) an amplified tunable laser (maximum $\mathrm{CW}$ power $1 \mathrm{~W}$, tuning range $1530-1565 \mathrm{~nm}, \mathrm{SNR}>50 \mathrm{~dB}$, linewidth $<20 \mathrm{pm}$ ), and (b) a monomode fiber-coupled diode laser centered at $975 \mathrm{~nm}$ (maximum power $500 \mathrm{~mW}$ ). During our measurements no significant change of linewidth or line shape was observed, so we concluded that no transverse gradient of temperature was produced.

The thermal profiles of uniformly irradiated sections of different fibers were measured in detail. Figure 2 shows the characterization of fiber PS980 (irradiated section: $5 \mathrm{~mm}$ ). The UV-fluence used in the irradiation process was $150 \mathrm{~J} / \mathrm{mm}^{2}$ in a single sweep. Figure 2(a) shows the results when the fiber was illuminated with the $1550 \mathrm{~nm}$ optical signal (1 W optical power), while Fig. 2(b) depicts the measurements for the $975 \mathrm{~nm}$ laser (500 $\mathrm{mW}$ optical power). The results show a clear difference between the irradiated section $(z<3 \mathrm{~mm})$ and the pristine fiber $(z>3 \mathrm{~mm})$. For $z>3 \mathrm{~mm}$, it can be observed a spacial transient governed by the temperature gradient, the heat conduction in silica and the heat transfer to the air. The maximum heating rate obtained when illuminating at $1550 \mathrm{~nm}$ is higher than $10^{\circ} \mathrm{C} / \mathrm{W}$, while it is lower than $1^{\circ} \mathrm{C} / \mathrm{W}$ at $975 \mathrm{~nm}$, due to the higher absorption of the irradiated fiber at longer wavelengths. No significant difference in the heating was observed within the range $1530-1565 \mathrm{~nm}$.

A direct measurement of transmission loss variation was carried out for fiber PS980, while it was irradiated. First, the loss of the pristine PS980 fiber was measured at $1550 \mathrm{~nm}$ by means of the cutback method: a value of $120.0 \pm 0.5 \mathrm{~dB} / \mathrm{km}$ was obtained. Then the UV laser was swept back and forth along a 5 $\mathrm{cm}$ section of the fiber, repeatedly (energy delivered: $2.5 \mathrm{~J} / \mathrm{mm}^{2}$ per swept). The light provided by a LED source (spectral range: $1520-1560 \mathrm{~nm}$ ) was launched to the fiber, and the output was measured after each swept of the UV laser. The results are shown in Fig. 3(a). The final loss was $6.2 \pm 0.4 \mathrm{~dB} / \mathrm{m}$ (total energy delivered to the fiber: $160 \mathrm{~J} / \mathrm{mm}^{2}$ ). This means that the ratio of 

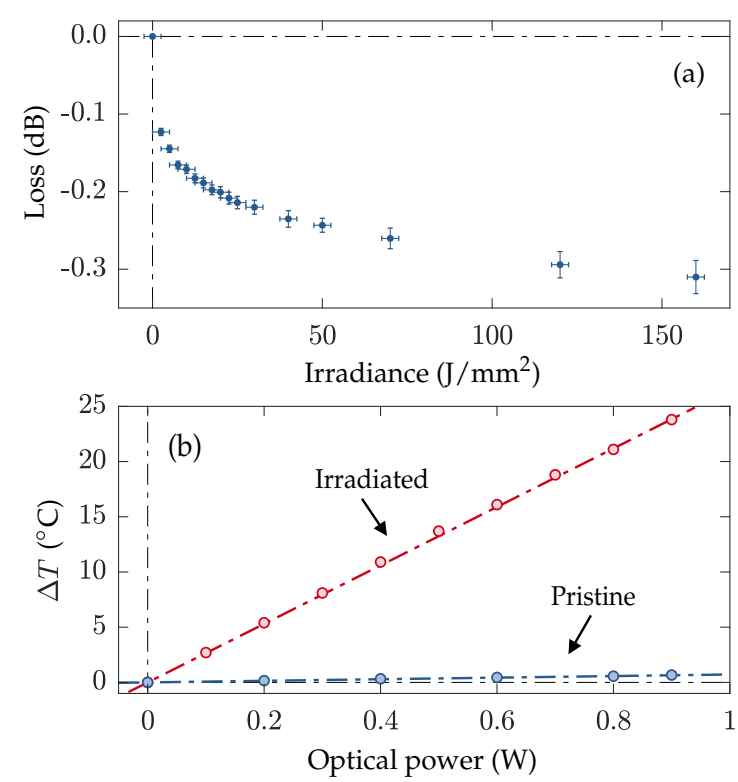

Fig. 3. (a) Direct measurement of the loss as the fiber is irradiated. Length: $5 \mathrm{~cm}$. (b) Thermal heating of the PS980 fiber as a function of the optical power of the input signal $(1550 \mathrm{~nm})$.

the attenuation coefficient, $\alpha_{2} / \alpha_{1}$, was increased in $52 \pm 3$ times. It should be noted that this attenuation coefficient includes the contribution of absorption and scattering $\left(\alpha=\alpha^{\text {abs }}+\alpha^{\text {scat }}\right)$.

The same section of the fiber was used to measure the increase of the absorption coefficient due to the UV illumination, by means of the thermal shift of WGM resonances. The $1550 \mathrm{~nm}$ laser signal was launched to the microresonator, and the wavelength shift of the resonances was measured as the power was increased. Results for the temperature variations are shown in Fig. 3(b). The data show a linear trend of the heating with the power of the signal, within and outside of the irradiated section. The temperature of the irradiated section increased at a rate of $26.48 \pm 0.15^{\circ} \mathrm{C} / \mathrm{W}$, and at $0.718 \pm 0.014{ }^{\circ} \mathrm{C} / \mathrm{W}$ in the pristine fiber. The ratio between both slopes is $36.9 \pm 0.7$. Thus, according to Eq. 2, the absorption coefficient is increased in this factor.

The same set of measurements was repeated for fibers PS1250, hydrogenated SMF28, and SM1500, at $1550 \mathrm{~nm}$. The results are compiled in Table 1 . It has to be taken into account that,

Table 1. Measurement of thermal heating and loss coefficient of different fibers

\begin{tabular}{|c|c|c|c|c|}
\hline & \multicolumn{2}{|c|}{$\begin{array}{c}\text { WGM resonances } \\
\Delta T / P\left({ }^{\circ} \mathrm{C} / \mathrm{W}\right)\end{array}$} & \multicolumn{2}{|c|}{$\begin{array}{l}\text { Direct measurement } \\
\qquad \alpha(\mathrm{dB} / \mathrm{km})\end{array}$} \\
\hline & Irradiated & Pristine & Irradiated & Pristine \\
\hline PS980 & $26.48 \pm 0.15$ & $0.718 \pm 0.014$ & $6200 \pm 400$ & $120 \pm 2^{(1)}$ \\
\hline PS1250 & $30.80 \pm 0.17$ & $0.768 \pm 0.014$ & $6600 \pm 400$ & $131.1^{(2)}$ \\
\hline $\mathrm{H}_{2}-\mathrm{SMF} 28$ & $23.48 \pm 0.13$ & $0.815 \pm 0.012$ & $5600 \pm 400$ & $\mathrm{n} / \mathrm{a}^{(3)}$ \\
\hline SM1500 & $1.20 \pm 0.03$ & $<0.03^{(4)}$ & $370 \pm 90$ & $1.95^{(2)}$ \\
\hline \multicolumn{5}{|c|}{$\begin{array}{l}\text { (1) Cutback measurement } \\
\text { (2) Nominal value } \\
\text { (3) Non-available, hydrogenated fiber } \\
\text { (4) Below detection limit }\end{array}$} \\
\hline
\end{tabular}

Table 2. Comparison between techniques

\begin{tabular}{|c|c|c|}
\hline & $\begin{array}{l}\text { WGM resonances } \\
\qquad \alpha_{2}^{\mathrm{abs}} / \alpha_{1}^{\mathrm{abs}}\end{array}$ & $\begin{array}{l}\text { Direct measurement } \\
\qquad \alpha_{2} / \alpha_{1}\end{array}$ \\
\hline PS980 & $36.9 \pm 0.7$ & $52 \pm 3$ \\
\hline PS1250 & $40.1 \pm 0.8$ & $50 \pm 3$ \\
\hline $\mathrm{H}_{2}-\mathrm{SMF} 28$ & $28.8 \pm 0.5$ & $\mathrm{n} / \mathrm{a}^{(1)}$ \\
\hline SM1500 & $>40^{(2)}$ & $190 \pm 50$ \\
\hline
\end{tabular}

while the WGMs technique allows measuring the absorption coefficient exclusively, the transmission technique also accounts for scattering loss that may happen along the fiber. The comparison between the two techniques is shown in Table 2: the

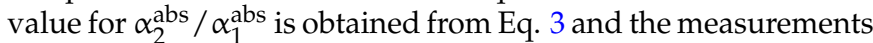
of the heating of the fibers using WGMs, while the ratio $\alpha_{2} / \alpha_{1}$ is calculated from direct transmission measurements of the irradiated fibers.

From these results (Tables 1 and 2), we can stablish some interesting conclusions for the UV effects on the fiber loss. Certainly, the absorption coefficient is substantially increased by a factor between 20 and 40 . This is what one could expect and it is consistent with the change of the refractive index that is exploited for the inscription of the fiber gratings. It is worth to point out that the actual values of $\Delta T / P$ demonstrate that in most medium and high power systems the temperature increase will be not negligible. For example, FBGs fabricated with these photosensitive fibers, using a typical value of $\sim 160 \mathrm{~J} / \mathrm{mm}^{2} \mathrm{UV}$ fluence, will exhibit important shifts and chirps [15].

In addition, our results show that the scattering losses are increased even more than the absorption losses. This is the only conclusion compatible with the results summarized in the Table 2 , where we see that $\alpha_{2} / \alpha_{1}$ is systematically higher than $\alpha_{2}^{\text {abs }} / \alpha_{1}^{\text {abs }}$. If we rewrite $\alpha$ as $\alpha^{\text {abs }}+\alpha^{\text {scat }}$ and we define $r$ as the ratio between $\alpha_{2} / \alpha_{1}$ and $\alpha_{2}^{\text {abs }} / \alpha_{1}^{\text {abs }}$, then we can find the following relationships:

$$
\begin{aligned}
& \frac{\alpha_{2}^{\mathrm{abs}}}{\alpha_{2}}=\frac{\alpha_{1}^{\mathrm{abs}}}{\alpha_{1}} \frac{1}{r} \\
& \frac{\alpha_{2}^{\text {scat }}}{\alpha_{2}}=\frac{\alpha_{1}^{\text {scat }}}{\alpha_{1}}+\frac{\alpha_{1}^{\mathrm{abs}}}{\alpha_{1}} \frac{r-1}{r}
\end{aligned}
$$

Equation 4 shows that when $r>1$, then the relative contribution of the absorption coefficient decreases after UV radiation, i.e., $\left(\alpha_{2}^{\text {abs }} / \alpha_{2}\right)<\left(\alpha_{1}^{\text {abs }} / \alpha_{1}\right)$. Furthermore, Eq. 5 shows that if $r>1$, then the relative contribution of scattering to the overall loss increase since the last term of the Eq. 5 is positive, i.e., $\left(\alpha_{2}^{\text {scat }} / \alpha_{2}\right)>\left(\alpha_{1}^{\text {scat }} / \alpha_{1}\right)$. If we divide Eq. 5 by Eq. 4, then we can show even more clearly how much the relative contribution of scattering is increasing with respect the absorption part for a ratio $r>1$ :

$$
\frac{\alpha_{2}^{\text {scat }}}{\alpha_{2}^{\mathrm{abs}}}=\frac{\alpha_{1}^{\text {scat }}}{\alpha_{1}^{\mathrm{abs}}} \cdot r+(r-1)
$$


Table 3. Absorption and scattering contributions to the overall attenuation coefficient

\begin{tabular}{ccccc}
\hline & \multicolumn{2}{c}{$\alpha^{\text {abs }}(\mathbf{d B} / \mathbf{k m})$} & \multicolumn{2}{c}{$\alpha^{\text {scat }}(\mathbf{d B} / \mathbf{k m})$} \\
\cline { 2 - 5 } & Irradiated & Pristine & Irradiated & Pristine \\
\hline PS980 & $3680 \pm 20$ & $99.7 \pm 1.9$ & $2500 \pm 400$ & $20 \pm 3$ \\
\hline PS1250 & $4280 \pm 20$ & $106.6 \pm 1.9$ & $2300 \pm 400$ & $24.5 \pm 1.9$ \\
\hline $\mathrm{H}_{2}$-SMF28 & $3260 \pm 18$ & $113.1 \pm 1.7$ & $2300 \pm 400$ & $\mathrm{n} / \mathrm{a}^{(1)}$ \\
\hline SM1500 & $167 \pm 4$ & $<1.95^{(2)}$ & $200 \pm 90$ & $<1.95^{(2)}$ \\
\hline
\end{tabular}

(1) Non-available, hydrogenated fiber

(2) Nominal value

The above discussion has been carried out using experimental measurements and Eq. 3. So the conclusions are virtually free of any heat dissipation model, but limited to the evaluation of relative values. However, if we now take into account Eq. 2 and the values of $h$ reported in [12], then we can obtain absolute values for the absorption and scattering contributions to the overall attenuation coefficient. Table 3 summarizes the numerical values that we obtained for $\alpha^{\text {abs }}$ using the experimental slopes $\Delta T / P$ and Eq. 2, and the values computed for $\alpha^{\text {scat }}\left(\alpha^{\text {scat }}=\alpha-\alpha^{\text {abs }}\right)$.

Following this procedure, we are able to provide the two independent contributions $\alpha^{\text {abs }}$ and $\alpha^{\text {scat }}$ to the overall attenuation coefficient. We can see that in the UV-irradiated fibers both contributions are of the same order of magnitude, even though $\alpha^{\text {scat }}$ is smaller, between 0.53 and 0.70 times $\alpha^{\text {abs, }}$, excepting fiber SM1500 in which $\alpha^{\text {scat }}$ is 1.19 times $\alpha^{\text {abs }}$. In the case of pristine fibers PS980 and PS1250, we can evaluate the two contributions and we see that $\alpha^{\text {scat }}$ is between 0.20 and 0.23 times $\alpha^{\text {abs }}$. This confirms that the UV radiation produces an increase of the scattering loss faster than the absorption loss.

The technique based on WGM resonances presents an additional advantage, due to its axial resolution. The direct measurement of the UV-induced loss provides an average result over the length of the section of the fiber under test (in our case, $5 \mathrm{~cm}$ ), while WGM resonances enables point to point measurements. Thus, it is possible to use this technique in order to study the uniformity of the absorption coefficient (and, hence, both the UV-irradiation and the refractive index variation) along the section of fiber. As a consequence, our technique can be used to detect and correct smooth undesired non-homogeneities or chirps introduced during the fabrication of gratings, taking into

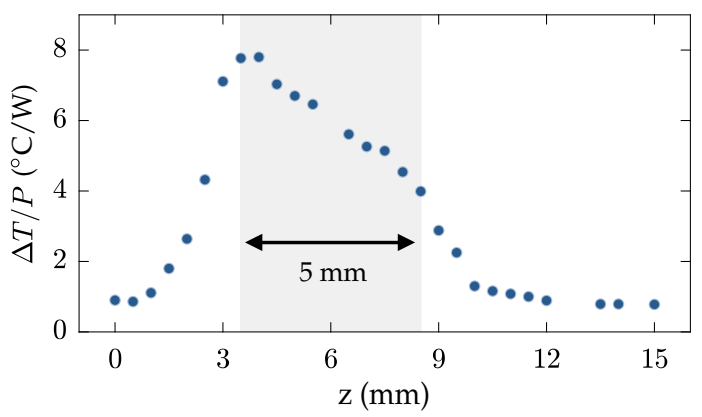

Fig. 4. Thermal profile of an irradiated section of PS980 with a non-uniform UV irradiation along the length of the fiber. account that the axial resolution of the technique will be larger than the grating period. As a practical example, Fig. 4 shows the thermal profile of a fiber that suffered some misalignment during the UV-irradiation process. The length of the irradiated fiber was $5 \mathrm{~mm}$, and the nominal energy from the UV-laser was $150 \mathrm{~J} / \mathrm{mm}^{2}$ in a single sweep. The usual input signal $(1 \mathrm{~W}$ at $1550 \mathrm{~nm}$ ) was employed. It can be observed that the WGM resonances technique allows identifying and quantifying the different heating along sections of just a few $\mathrm{mm}$, which corresponds to different absorptions coefficients and refractive index increments.

We conclude that the technique presented in this work enables a direct characterization of absorption losses and the uniformity of UV-irradiation in photosensitive fibers. By combining our results with conventional measurements of the fiber attenuation, we can evaluate separately the absorption and scattering contributions to the overall losses. In our experiment, the scattering losses increase faster than the absorption losses under UV-irradiation, although they remain in general lower. We find that our technique might be useful not only for further improvements of photosensitive fibers characteristics, but for the characterization of active and specially doped fibers. For example, it should be possible to study if a specific doping technique increases the scattering losses unnecessarily, but not so much the absorption.

Funding: Ministerio de Economía y Competitividad of Spain and FEDER funds (Ref: TEC2016-76664-C2-1-R); Generalitat Valenciana (Ref: PROMETEOII/2014/072), Universitat de València (UV-INV-AE16-485280). X. Roselló-Mechó's contract is funded by the FPI programme (MinECo, Spain, BES-2014-068607).

\section{REFERENCES}

1. R. Kashyap, "Photosensitivity and photosensitization of optical fibers," in "Fiber Bragg Gratings," R. Kashyap, ed. (Academic Press, San Diego, 1999), Optics and Photonics, pp. $13-54$.

2. A. Arigiris, M. Konstantaki, A. Ikiades, D. Chronis, P. Florias, K. Kallimani, and G. Pagiatakis, Opt. Lett. 27, 1306 (2002).

3. M. Gagné, S. Loranger, J. Lapointe, and R. Kashyap, Opt. Express 22, 387 (2014).

4. B. Poumellec, P. Niay, M. Douay, and J. F. Bayon, J. Phys. D: Appl. Phys. 29, 1842 (1996).

5. L. Dong, J. L. Cruz, L. Reekie, M. G. Xu, and D. N. Payne, IEEE Photonics Technol. Lett. 7, 1048 (1995).

6. B. Poumellec, P. Guénot, I. Riant, P. Sansonetti, P. Niay, P. Bernage, and J. Bayon, Opt. Mater. 4, 441 (1995).

7. J. Canning, M. Åslund, and P.-F. Hu, Opt. Lett. 25, 1621 (2000).

8. E. Rivera-Pérez, I. L. Villegas, A. Díez, M. V. Andrés, J. L. Cruz, and A. Rodríguez-Cobos, IEEE Photonics Technol. Lett. 25, 2498 (2013).

9. M. Sumetsky and Y. Dulashko, Opt. Lett. 35, 4006 (2010).

10. T. A. Birks, J. C. Knight, and T. E. Dimmick, IEEE Photonics Technol. Lett. 12, 182 (2000).

11. X. Roselló-Mechó, M. Delgado-Pinar, A. Díez, and M. V. Andrés, Opt. Lett. 41, 2934 (2016).

12. M. K. Davis, M. J. F. Digonnet, and R. H. Pantell, J. Light. Technol. 16, 1013 (1998).

13. M. Delgado-Pinar, I. L. Villegas, A. Díez, J. L. Cruz, and M. V. Andrés, Opt. Lett. 39, 6277 (2014).

14. M. Sumetsky, Opt. Lett. 35, 2385 (2010).

15. J. Lauzon, S. Thibault, J. Martin, and F. Ouellette, Opt. Lett. 19, 2027 (1994). 


\section{FULL REFERENCES}

1. R. Kashyap, "Photosensitivity and photosensitization of optical fibers," in "Fiber Bragg Gratings," R. Kashyap, ed. (Academic Press, San Diego, 1999), Optics and Photonics, pp. $13-54$.

2. A. Arigiris, M. Konstantaki, A. Ikiades, D. Chronis, P. Florias, K. Kallimani, and G. Pagiatakis, "Fabrication of high-reflectivity superimposed multiple-fiber Bragg gratings with unequal wavelength spacing," Opt. Lett. 27, 1306-1308 (2002).

3. M. Gagné, S. Loranger, J. Lapointe, and R. Kashyap, "Fabrication of high quality, ultra-long fiber Bragg gratings: up to 2 million periods in phase," Opt. Express 22, 387-398 (2014).

4. B. Poumellec, P. Niay, M. Douay, and J. F. Bayon, "The UV-induced refractive index grating in $\mathrm{Ge}: \mathrm{SiO}_{2}$ preforms: additional $\mathrm{CW}$ experiments and the macroscopic origin of the change in index," $\mathrm{J}$. Phys. D: Appl. Phys. 29, 1842 (1996).

5. L. Dong, J. L. Cruz, L. Reekie, M. G. Xu, and D. N. Payne, "Enhanced photosensitivity in tin-codoped germanosilicate optical fibers," IEEE Photonics Technol. Lett. 7, 1048-1050 (1995).

6. B. Poumellec, P. Guénot, I. Riant, P. Sansonetti, P. Niay, P. Bernage, and J. Bayon, "UV induced densification during Bragg grating inscription in $\mathrm{Ge}: \mathrm{SiO}_{2}$ preforms," Opt. Mater. 4, 441 - 449 (1995).

7. J. Canning, M. Åslund, and P.-F. Hu, "Ultraviolet-induced absorption losses in hydrogen-loaded optical fibers and in presensitized optical fibers," Opt. Lett. 25, 1621-1623 (2000).

8. E. Rivera-Pérez, I. L. Villegas, A. Díez, M. V. Andrés, J. L. Cruz, and A. Rodríguez-Cobos, "Measurement of pump-induced temperature increase in doped fibers using whispering-gallery modes," IEEE Photonics Technol. Lett. 25, 2498-2500 (2013).

9. M. Sumetsky and Y. Dulashko, "Radius variation of optical fibers with angstrom accuracy," Opt. Lett. 35, 4006-4008 (2010).

10. T. A. Birks, J. C. Knight, and T. E. Dimmick, "High-resolution measurement of the fiber diameter variations using whispering gallery modes and no optical alignment," IEEE Photonics Technol. Lett. 12, 182-183 (2000).

11. X. Roselló-Mechó, M. Delgado-Pinar, A. Díez, and M. V. Andrés, "Measurement of Pockels' coefficients and demonstration of the anisotropy of the elasto-optic effect in optical fibers under axial strain," Opt. Lett. 41, 2934-2937 (2016).

12. M. K. Davis, M. J. F. Digonnet, and R. H. Pantell, "Thermal effects in doped fibers," J. Light. Technol. 16, 1013-1023 (1998).

13. M. Delgado-Pinar, I. L. Villegas, A. Díez, J. L. Cruz, and M. V. Andrés, "Measurement of temperature profile induced by the optical signal in fiber Bragg gratings using whispering-gallery modes," Opt. Lett. 39, 6277-6280 (2014).

14. M. Sumetsky, "Mode localization and the Q-factor of a cylindrical microresonator," Opt. Lett. 35, 2385-2387 (2010).

15. J. Lauzon, S. Thibault, J. Martin, and F. Ouellette, "Implementation and characterization of fiber Bragg gratings linearly chirped by a temperature gradient," Opt. Lett. 19, 2027-2029 (1994). 